\title{
Enhanced Volume of Stem Cell-Based Regenerated Tissue Achieved by Altering Microenvironment and Cell Seeding Strategies
}

\author{
Ricardo A Rosselló ${ }^{1,2,4^{*}}$ and David H Kohn ${ }^{2,3}$ \\ ${ }^{1}$ Departments of Biochemistry, University of Puerto Rico, Medical Sciences Campus, San Juan, Puerto Rico \\ ${ }^{2}$ Departments of Biomedical Engineering, University of Michigan, Ann Arbor, MI, USA \\ ${ }^{3}$ Departments of Biologic and Materials Sciences, University of Michigan, Ann Arbor, MI, USA \\ ${ }^{4}$ Department of Science and Technology, Metropolitan University, San Juan, Puerto Rico
}

*Corresponding author: Ricardo A Rossello, Department of Biochemistry, University of Puerto Rico, Medical Sciences Campus, San Juan, Puerto Rico, Tel: +1 787-754-0101; E-mail: ricardo.rossello@upr.edu

Received date: October 27, 2013; Accepted date: April 20, 2014; Published date: April 23, 2014

Copyright:@ 2014 Rossello AR, et al. This is an open-access article distributed under the terms of the Creative Commons Attribution License, which permits unrestricted use, distribution, and reproduction in any medium, provided the original author and source are credited.

\begin{abstract}
Tissue engineering has emerged as a potential alternative or complementary solution to organ failure or damage. Adult stem cell based approaches can provide a powerful platform for regeneration, given these cells capacity to differentiate into multiple tissues, given the appropriate signals. However, a barrier in the development of tissue in $3 \mathrm{D}$ constructs is the transport limitations of nutrients and by products to the core, producing low volumes of regenerated tissue. The microenvironment in the immediate vicinity of the cells, the number of cells that adhere to the substrate and their localization may play an important role in differentiation and tissue regeneration. In this study, we aim to overcome compromised development of hard tissue using 3D cell-based tissue engineering strategies. Specifically, we quantified the effect of a biomimetic template and cell seeding techniques in Gap Junction Intercellular Communication (GJIC), differentiation (Osteocalcin and Alkaline Phosphatase mRNA) In-vitro and bone volume fraction (In-vivo) of adult stem cells (Bone Marrow Stromal Cells) seeded in 3D rigid scaffolds. Significant increases in amount and distribution of bone were achieved when altering both the template and initial seeding conditions. Our findings indicate that creating a biomimetic environment and altering initial seeding conditions that enhance cell adhesion and cell-cell communication in rigid scaffolds are powerful strategies to overcome the incomplete regeneration of cell-based engineered tissue.
\end{abstract}

Keywords: Bone; Cell seeding; Tissue engineering; Biomimetics; Gap junctions

\section{Introduction}

Initial conditions may play an important role in the ultimate result of tissue regeneration. For example, by overcoming signaling and transport limitations that cell in the interior of tissue equivalents experience, larger and more uniform volumes of tissue can be regenerated [1]. A particular challenge are skeletal defects, which present a major clinical challenge with over 5.5 million fractures and 1 million bone grafting procedures done each year [2]. Present therapies, such as allogenic bone transplantation and non-bioactive materials have limitations that include an adverse immune response and incomplete regeneration of tissue, respectively [3]. An alternative to these grafting techniques and materials is a cell transplantation approachwhere 3-dimensional synthetic constructs provide a biomimetic substrate for cells to regenerate tissue engineered bone $[4,5]$. These substrates are designed to enhance cellular growth, differentiation, and provide a temporary template for the formation of extracellular matrix and new tissue [6-10]. A mineralized layer on the surface of a porous 3D scaffold can provide a physiologically favorable environment that enhances cell adhesion and osteoconductivity $[11,12]$. However, this approach too has limitations due to initial cell seeding retention, as well as diminished transport of nutrients, signals, and messengers [13-15]. Such limitations in bone growth are largely caused by cells that thrive and differentiate quickly in the periphery of the construct that is exposed to nutrients and signals, while cells in the core of the construct are then left with a diminished capacity to differentiate, inhibiting bone in-growth.

A potential strategy to overcome these limitations is to alter the initial cell seeding techniques to enhance nutrient transport, cell density, and cell-cell communication in 3D scaffold. Static seeding, a method by which cells are pipetted into the scaffold, suffers from low cell retention rates. Several different strategies have been used in-vitro to increase cell viability throughout a 3D construct. Perfusion systems that provide flow conditions within the $3 \mathrm{D}$ structures have been shown to enhance cell viability and nutrient exchange in a variety of cell culture tissues, including bone, muscle and liver [10,13,14]. Also, high density cell clusters have been studied in two-dimensional monolayer and have demonstrated an increased capacity to differentiate [7]. These two techniques may also address a particular interest in 3D cultures; the coupling of chemical and mechanical signals that are transferred intercellulary [16-18]. Gap junctions may serve as a potential tissue engineering tool to address the passage of such signals [1]. Composed of two juxtaposed hemichannels (connexins) present on the surfaces of adjacent cells, gap junctions form a transcellular channel that permits the rapid and efficient propagation of ions, metabolites, and second messengers between adjoining cells [19]. By increasing cell density, Gap Junction Intercellular Communication (GJIC) may be enhanced.

In this study, we hypothesize that a $3 \mathrm{D}$ rigid biomimetic template produces a favorable microenvironment for in-vivo regeneration of tissue, and that altering the initial seeding strategies by enhancing cell 
retention, will produce larger and spatially distributed tissue equivalents of bone. To achieve this, we first incubate PLGA scaffolds in Simulated Body Fluid (SBF), to enable surface mineralization, as has been previously performed [20]. Briefly, a bone-like apatite can form in the polymer scaffold and mimic physiological conditions similar to blood plasma [20,32]. In this case, the PLGA will function as a bulk material that degrades at a controlled rate while the mineral layer serves as a biological interface. Additionally, the surface mineralization is expected to provide a bioactive surface to moderate $\mathrm{Ca}++$ flux into the cells, for enhanced differentiation, signaling and cellular growth [12,21-24]. This calcium flux is an important cell-cell messenger in osteoblasts that may be important for proper bone remodeling and regeneration $[25,26]$.

To address the seeding density and localization problem observed in the static method, we propose two alternative seeding techniques: seeding cells by filtration and dense micromass seeding. Our filtration method circulates the cell suspension through the scaffolds, with a small pressure gradient applied by a peristaltic pump. The homogeneous cell suspension that filters through the scaffold is cycled through and may produce higher cell density and even cellular distribution, as it recycles the cells that are not attached and promotes adhesion in alternative sites. The increased cell density, nutrient flux and sheer stress may therefore produce higher cellular adhesion and lower variability among samples. Although higher density of cells is desired to increase the cell-cell contacts that enable cellular communication and signaling, it may produce an adverse microenvironment in the core of the scaffolds due to a super saturation of cells inhibiting transport [27]. Passive nutrient diffusion is one of the biggest obstacles in $3 \mathrm{D}$ cell-scaffold composite systems [28]. Particularly for bone, the outer layer of cells and tissue prohibits proper exchange of nutrients and byproducts inside the ossicle's core. One way to bypass this is by seeding cells in dense micromasses. These micromasses are placed in a particular location in the scaffold (center), leaving less crowded areas were nutrients can flow through. Micromasses could provide the benefits of higher density cell-cell communication, while allowing for transport of nutrients and cellular byproducts, as well as migration of cells that would enable a differentiation gradient from the core to the periphery.

Our experiments aim to answer two critical questions in hard tissue engineering. First, we propose that by altering seeding strategies we can increase the cell retention, cell-cell communication, and differentiation in rigid 3D scaffolds. Secondly, we examine the effects of changing the initial template and cell seeding on the amount and distribution of regenerated bone in-vivo. The broad implications of this study may impact all facets of 3D in-vivo tissue engineering.

\section{Materials and Methods}

\section{Bone marrow stromal cell (BMSCs) isolation and culture}

Five-week old C57BL/6 mice were used to isolate bone marrow cells from the femoral, tibial and humeral cavities (six bones per animal) as previously described [29]. Briefly, the bone marrow was mixed with minimum essential medium ( $a-M E M$; Gibco Laboratories, Grand Island, NY) containing 10\% fetal bovine serum (FBS) (Gibco) and antibiotics $(100 \mu \mathrm{g} / \mathrm{ml}$ penicillin $\mathrm{G}$ and $100 \mathrm{IU} / \mathrm{ml}$ streptomycin at $37^{\circ} \mathrm{C}$ in $5 \% \mathrm{CO}_{2} / 95 \%$ air). Cells were pelleted by centrifugation at 1000 rpm for $5 \mathrm{~min}$ at $4^{\circ} \mathrm{C}$ and resuspended in $10 \mathrm{ml} \mathrm{a}$-MEM. Cells were plated at a density of 30,000 nucleated cells $/ \mathrm{cm}^{2}$, and cultured under the same conditions. The culture medium was replaced three times per week and at near confluence (90\%) the adherent cells were washed with phosphate-buffered saline solution and enzymatically released by means of a $0.25 \%$ trypsin-EDTA solution (Sigma, St. Louis, MO). Cells were re-plated at a density of 30,000 cells $/ \mathrm{cm}^{2}$ and subsequent passages were performed 7-10 days after, when cells achieved near confluence. Cells were passaged twice before they were used in the subsequent experiments.

\section{Control culture of mouse fibroblasts}

Embryonic fibroblasts were collected at embryonic day 12.5 for mice. Briefly, several embryos of C57BLK mice $(n=4)$ were extracted from the womb or eggs, their head, limbs, and liver removed, and the remaining contents were minced manually using forceps. The minced contents were placed in a $15 \mathrm{ml}$ tube and treated with $0.25 \%$ trypsin (0.25\% Trypsin/EDTA, Gibco; $1-2 \mathrm{ml}$ per embryo) for $30 \mathrm{~min}$ at $37^{\circ} \mathrm{C}$, pipetting briefly every 5 minutes to enhance dissociation. Trypsin was neutralized with complete media, cells were spun down, counted (hemocytometer), re-suspended in complete media and plated at a concentration of one embryo per $150 \mathrm{~mm}$ dish for mouse and per 100 $\mathrm{mm}$. When grown to confluent layers, all fibroblasts were passaged in complete media twice before cells were frozen in aliquots.

\section{Scaffold preparation}

Porous, 3D organic templates (85:15 poly(lactide-co-glycolide), diameter $=4 \mathrm{~mm} \times$ height $=1 \mathrm{~mm}, 90 \%$ porosity, pore size $250-425$ $\mu \mathrm{m})$ were prepared by a solvent particulate leaching processexplained elsewhere [20,30,31]. Briefly, PLGA and sodium chloride particles are mixed and cast into a particular geometry, through which a liquid could pass through. Finally, water was passed through the mold to "leach out" the salt particles so as to create a porous structure.

\section{Mineralization of Scaffolds}

Scaffolds were each incubated in a $50 \mathrm{~mL}$ solution of modified Simulated Body Fluid (SBF) for 7 days for mineral film formation [32]. The SBF solution was changed every $24 \mathrm{~h}$ to ensure sufficient ion concentrations for mineral growth. The SBF was prepared by dissolving the following reagents in deionized water: $141 \mathrm{mM} \mathrm{NaCl}$, $4.0 \mathrm{mM} \mathrm{KCl}, 0.5 \mathrm{mM} \mathrm{MgSO}_{4}, 1.0 \mathrm{mM} \mathrm{MgCl}$, $4.2 \mathrm{mM} \mathrm{NaHCO}_{3}, 5.0$ $\mathrm{mM} \mathrm{CaCl}_{2}$, and $2.0 \mathrm{mM} \mathrm{KH}_{2} \mathrm{PO}_{4}$. SBF was buffered to maintain a $\mathrm{pH}$ 7.4 with Tris $-\mathrm{HCl}$ at $37^{\circ} \mathrm{C}$ for the duration of the incubation period.

\section{Pre-wetting scaffolds}

After mineralization, scaffolds were pre-wetted with $70 \%$ ethanol by pressing wet pads around the surface area for 5 minutes. Afterwards, scaffolds were submerged in $50 \mathrm{ml}$ falcon tubesfilled with a-MEM and agitated 30 minutes to remove the excess ethanol. The scaffoldswere removed from the tube and placed into a new one with fresh a-MEM. The process was done 5-6 times until the $\mathrm{pH}$ of the $\alpha$-MEM in the tube matched that of the sterile $a-M E M(p H=7.1)$. The scaffold was then left to soak in the media overnight before seeding.

\section{Cell seeding}

The experimental design assessed three methods of seeding (static, dynamic, and filtration) at three time points ( $1 \mathrm{hr}, 6 \mathrm{hrs}$, and $24 \mathrm{hrs}$ ), using both mineralized and non-mineralized PLGA scaffolds. 6 scaffolds were used for each procedure, for a given time point and material. Static seeding was performed in 24 -well plates by pipetting a 
Page 3 of 11

cell suspension into the scaffold. Each well contained 1 scaffold. A cell suspension of $1 \mathrm{ml}$ with a density of $0.8 \mathrm{EE} 6 \mathrm{cells} / \mathrm{ml}$ was used in all the experiments. The scaffolds were placed into the incubator (at $37^{\circ} \mathrm{C}$ in $5 \% \mathrm{CO}_{2} / 95 \%$ air for all seeding methods) immediately after. For a perfusion type approach, we designed both dynamic and filtration seeding methods. Dynamic seeding was performed by trapping two scaffolds in a $15 \mathrm{ml}$ falcon tube, between meshes, with a stir bar outside the meshes in the bottom of the tube. The cell suspension was poured into the tubes, and these were placed in a stir plate inside an incubator. The stir intensity was set at $150 \mathrm{rpm}$. In filtration seeding 4 scaffolds were placed in 4 glass cylinders of the same radius. This method circulates the cell suspension through the scaffolds, with a small gradient applied by a peristaltic pump $(1.37 \mathrm{ml} / \mathrm{min})$ through small $(\mathrm{ID}=4 \mathrm{~mm}$ ) non-stick tubes. A homogeneous cell suspension is kept by adding a stir bar to the suspension reservoir. Scaffolds $(D=4$ $\mathrm{mm}, \mathrm{h}=1 \mathrm{~mm}$ ) were placed in a cylindrical scaffold chamber (ID $=4$ $\mathrm{mm}, 20 \mathrm{~mm}$ ). The chamber is non-stick so that the cells won't attach to its surface and only attach to the scaffolds. Based on the viscosity and density, the length of the tubes was estimated to be $0.3 \mathrm{~m}$, in order to generate a laminar profile and keep the $\mathrm{pH}$ at 7.4. The complete system was placed in a $\mathrm{CO}_{2}$ incubator, to promote gas exchange through the tubes. Cells that are not seeded in the first filtrate are passed through several times until maximum retention is reached. By experimental design, the perfusion method that would achieve higher cell retention would be selected for biological experimentation. In Micromass seeding, cells were pelleted in $5 \mathrm{X}$ concentration and pipetted, as in static seeding, into the middle of the scaffold (4.0 EE6 cells $/ \mathrm{ml})$.

\section{Cell counting and histology}

After the seeding experiment, the scaffolds were retrieved and washed with $\alpha$-MEM. The washed fraction was saved to count the free cells present in the media. Washing was done 5 times to retrieve all the free (unattached) cells. The apparatus and containers were also washed to determine the number of cells that did not adhere. Free cell count was obtained using a hemacytometer. The $\%$ of cells that adhered was determined by substracting washed cells from the original cell numbers. To verify this strategy, 4 scaffolds were treated with trypzinEDTA, to remove the attached cells. Trypsinization was performed for two minutes, followed by flushing of cells with a-MEM. This process was repeated 3 times per scaffold, and the resulting cell suspension was pooled to obtain the attached cell count using a hemacytometer. Attached cell counts from micromass seeded scaffolds were omitted as the retrieved cell count was inconsistent and the treatment destroyed some cells. A 3-way ANOVA on time, template, and seeding strategy was performed to determine significant differences $(p<0.005)$. Three scaffolds for each seeding strategy and time were analyzed histologically to quantify cell retention per sections and qualitatively observe the distribution of attached cells. These scaffolds were placed in $10 \%$ buffered formalin and ethanol. $5 \mu \mathrm{m}$ sections were made and placed on 10 slides with 3 sections per slide. Sections were standardized for all scaffolds in $200 \mu \mathrm{m}$ increments from the surface. The number of cells per section was quantified and the mean number of attached cells per section was reported. Differences were assessed using a 1-way ANOVA on 6 hour sections and significant differences were assessed at $\mathrm{p}>0.005$.

\section{Dye transfer studies}

Fluorescent dye transfer studies were performed to assess GapJunctional Intercellular Communication (GJIC) between BMSCs seeded in mineralized and non-mineralized scaffolds by static, filtered and micromass seeding strategies. The cell scaffold constructs $(4 \mathrm{~mm} \mathrm{x}$ $1 \mathrm{~mm}$ ) were seeded as previously described and placed in 24 well plates, to serve as recipient cells. Scaffolds were let in culture for one day. Calcein-AM (10 uM) and Vybrant-DiI were used to label donor cells grown to confluence in a 12-well plate. As a negative control, $50 \mathrm{uM}$ of the gap junction uncouplerAlpha-Glyccirrhetinic Acid (AGA) was used. Donor cells were added to potential recipient cells at 1:8 ratio. Cells were harvested by trypsinisation for quantitative assessment of GJIC by flow cytometry after 5 hours. The transfer regions for recipient cells ( $>2 \times 101$ Fl- 1 and $<5 \times 103$ Fl-1) and nonlabeled, non-recipient cells $(<2 \times 101$ Fl-1) were defined as the transfer regions based on the initial fluorescence range quantified fornonrecipient cells, and cells containing both dyes. Florescence above $5 \mathrm{x}$ 103 Fl-1 was indicative of cells containing both membrane tracker and calcenin-AM. This experiment was performed with $n=6$ groups. Statistical differences were measured using a 2-way ANOVA on template and seeding strategy $(\mathrm{p}<0.005)$.

\section{RTPCR Analysis of differentiation markers}

Fibroblasts and BMSCs were seeded in Mineralized and nonmineralized scaffolds by static, filtration and micromass strategies. The seeded constructs were placed in 24 well plates supplemented with osteogenic media (a-MEMmedia, 10\%Fetal Bovine Serum, 1\% Pen/ Strep, $1 \% 100 \mathrm{x} \beta$-Glycerophosphate, $1 \%$ L-ascorbic acid-phosphate, $\sim 0.05 \% 5000 x$ Dexamethasone). An additional group in which $1.5 \mathrm{mM}$ of $\mathrm{Ca}^{2+}$ was added to osteogenic media to assess for the effects of soluble calcium. Media was replenished every 24 hours. At 2, 8, and 16 days for analysis real-time PCR was used to detect the expression of two bone differentiation markers (Alkaline Phosphatase (ALP) and Osteocalcin $(\mathrm{OCN}))$. Primers and TaqMan probes were purchased (ABI). The primer sequences utilized were as follows: OCN, 5'CCAGCGACTCTGAGTCTGACAA-3', and 5'CCGGAGTCTATTCACCACCTTACT-3';ALP; 5 'GCCCTCTCCAAGACATATA-3' and $3^{\prime}$ CCATGATCACGTCGATATCC-3'

Seeded constructs weretrypzinized after 2, 8 and 16 days, to remove cells and the total RNA was extracted (Trizol; Invitrogen Corp). The RNA was purified (RNeasy, Quigen) and treated with DNAse I. Cycling conditions were as follows: $48^{\circ} \mathrm{C}$ for 10 minutes and $95^{\circ} \mathrm{C}$ for ten minutes, followed by 4 cycles of $95^{\circ} \mathrm{C}$ for 15 seconds and $60^{\circ} \mathrm{C}$ for 1 minute. No-template control analyses were run for each primer set and 18s rRNA endogenous control was run for each sample. The $2 \Delta \Delta \mathrm{CT}$ relative quantization method was utilized to evaluate gene expression. All reactions were performed in quintuplet and $n=4$. The results were normalized to the endogenous 18 s expression (ABI). A 3way ANOVA was used to determine significant differences as a function of seeding condition, scaffold, and time.

\section{Transplantation of cell-scaffold constructs}

Fibroblast and BMSCs were seeded by the static, filtration and micromass seeding as previously described. All cell-scaffold constructs were placed in an incubator for 1 hour (at $37^{\circ} \mathrm{C}$ in $5 \% \mathrm{CO}_{2} / 95 \%$ air) until transplantation. 24 Mineralized PLGA-BMSC and 24 PLGABMSC constructs were transplanted subcutaneously on nude mice 
$(\mathrm{nu} / \mathrm{nu})$. Each of the construct groups contained 6 filtered, 6 micromass, 6 static, 6 empty scaffolds. Briefly, nude mice (nu/nu) were anaesthetized by an intraperitoeal injection of $1 \mathrm{mg} / 10 \mathrm{~g}$ ketamine and $0.1 \mathrm{mg} / 10 \mathrm{~g}$ xylazine. An incision was made on the back of each mouse and the implants were inserted within the subcutaneous cavities. The wounds were closed with surgical clips aseptically. The mice were subsequently euthanized after 6 weeks to remove the regenerated bone ossicles.

\section{Micro-CT 3D image acquisition and analysis}

Ossicles were scanned on a high resolution cone beam micro-CT system (Enhanced Vision Systems (now GE Healthcare Preclinical Imaging), London, Ontario, Canada) while immersed in distilled $\mathrm{H}_{2} \mathrm{O}$. The $\mathrm{x}$-ray source voltage and current were $80 \mathrm{kVp}$ and $80 \mu \mathrm{A}$, respectively. To reduce the potential for beam hardening artifact, the $\mathrm{x}$-rays were passed through a $0.2 \mathrm{~mm}$ Al filter immediately upon exiting the source and the specimens were immersed in $\mathrm{dH}_{2} \mathrm{O}$ during the scanning process. Projection images were acquired over 198 degrees using $2 \times 2$ binning and an exposure time of $1100 \mathrm{~ms}$, and four frames were averaged for each projection to improve the signal to noise ratio. The projection data was then corrected and reconstructed using the Feldkamp cone-beam algorithm to create three-dimensional images with an isotropic voxel size of $18 \mu \mathrm{m}$. The scanner was calibrated once daily using a phantom that contained air, water and hydroxyapatite.

Bone volume fractions were determined by using a MatLab program designed to integrate all grayscale voxels above a particular threshold. To determine the overall volume of the ossicles, the program determined the perimeter of each $2 \mathrm{D} \mu \mathrm{CT}$ slice by tracing the outer edge. The program then integrated all the perimeters to determine the 3D surface area, and the number of voxels inside the surface defined the total volume. High density voxels outside of the 3D surface and unattached to the ossicle were discarded, while voxels inside were evaluated at the specified thresholds to determine the BVF, which was calculated as the number of voxels above the threshold relative to the total number of voxels. Using this method, a threshold of 1100 was used to re-construct a rendered image of the ossicles and determine their distribution [33].

\section{Histological analyses}

The ossicles were rinsed in water and then decalcified in $10 \%$ formic acid for 5 days. After decalcification, the tissues were embedded in paraffin. $5 \mu \mathrm{m}$ sections were made and placed on 10 slides with 3 sections per slide. The tissue was deparaffinized hydrated, and the first, fifth, and tenth slides were stained with $\mathrm{H} \& \mathrm{E}$, and von Kossa. Image Pro Plus 4.0 was used to take pictures of the histological sections.

\section{Analysis of bone ingrowth}

A program was developed to determine the distribution of regenerated bone as a function of the distance from the geometric center of each ossicle. Using von Kossa stained sections of bone ossicles regenerated in PLGA scaffolds, the centroid was calculated using a MatLab script and used as a frame of reference to divide the ossicles into 4 regions [34]. Defining the centroid as the 0th percentile and the edge as the 100th percentile, boundaries were calculated by lines that radially pointed into the center from the edges. Mineralized sections were determined by thresholding to the grayscale values represented by the dark von Kossa stain. Using this criterion, the program determined the percent of bone present in regions $0-25 \%$, $25-50 \%, 50-75 \%$ and $75-100 \%$ of the area away from the centroid. A 2way ANOVA was performed to differentiate between (1) sections in filtered and micromass generated ossicles and (2) topographical regions within each ossicle; significance was measured at $\mathrm{p}<0.005$.

\section{Results}

\section{Filtration seeding achieves a higher number of attached cells}

Filtration seeding led to significantly higher percentage of cells adhered than dynamic or static seeding (Figure 1).

PLGA

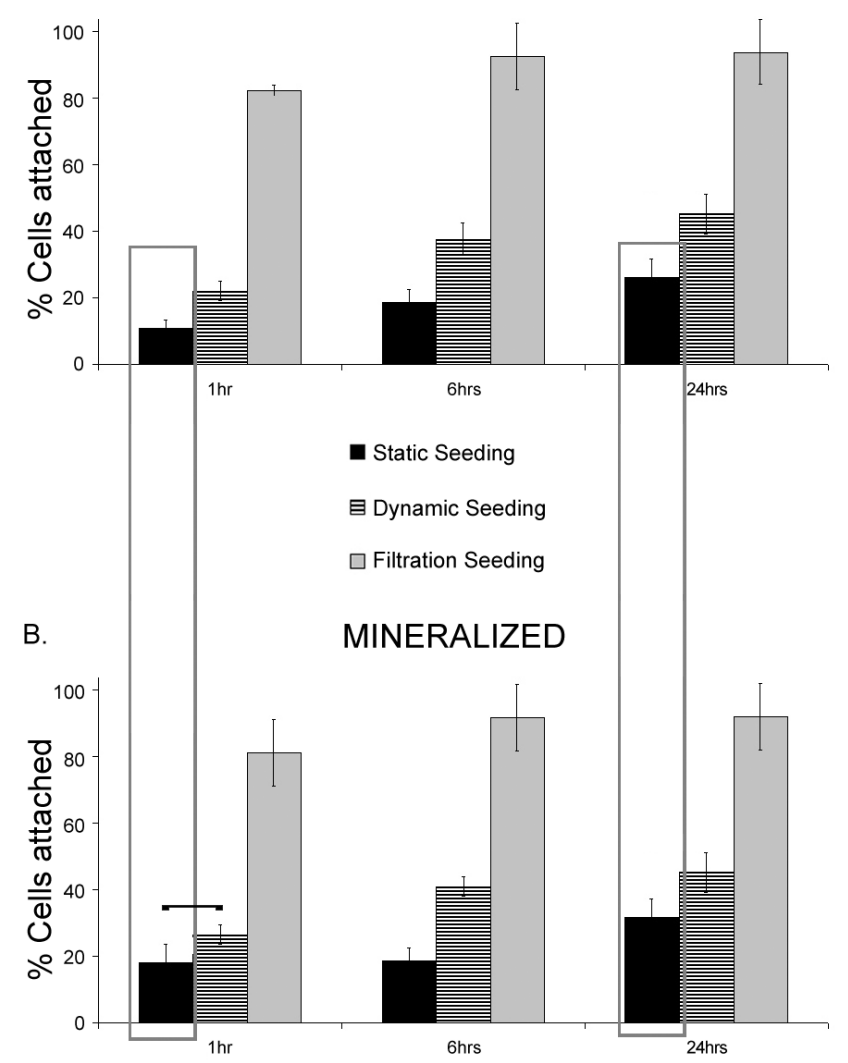

Figure 1: Percent of cells adhering to PLGA (A) and mineralized (B) scaffolds at different time points following seeding via different techniques. Using a filtration approach produces significantly more adhesion and cell retention than both dynamic and static seeding $(p<0.001)$. Filtration reaches a plateau after 6 hours. Both static and dynamic techniques increase the number of adhered cells as a function of time. There were no differences in adhesion between mineralized and PLGA scaffolds, with the exception of static seeding at 1 and 24 hours. Bars indicate pairs that were not significantly different. Boxes denote groups that showed significant difference in adhesion due to scaffold.

Scaffolds filtered with cells had high cell retention after 1 hour $(82.4+/-4.1 \%)$ and approached carrying capacity by the 6th hour 
$(92.32+/ 6.12)$. Both dynamic and static seeding increased as a function of time but had significantly less cell adhesion than filtration at all times ( $\mathrm{p}<0.001$, for all times). Dynamic also achieved significantly higher number of attached cells than static seeding $(\mathrm{p}(1 \mathrm{hr})=0.003$, $\mathrm{p}(6 \mathrm{hrs})<0.001, \mathrm{p}(24 \mathrm{hrs})=0.021)$. The effect of template was only significant in the static seeded scaffolds, where the mineralized layer enhanced adhesion $(\mathrm{p}(1 \mathrm{hr})=0.031, \mathrm{p}(6 \mathrm{hr})=0.028)$. There was no significant difference in cell adhesion between mineralized and nonmineralized scaffolds seeded by filtration and dynamic seeding. Histology verifies the adhesion results. The slides qualitatively show that filtration has an increase in cell number and spatial distribution when compared to static, dynamic, and micromass seeding (Figure 2A-2D).
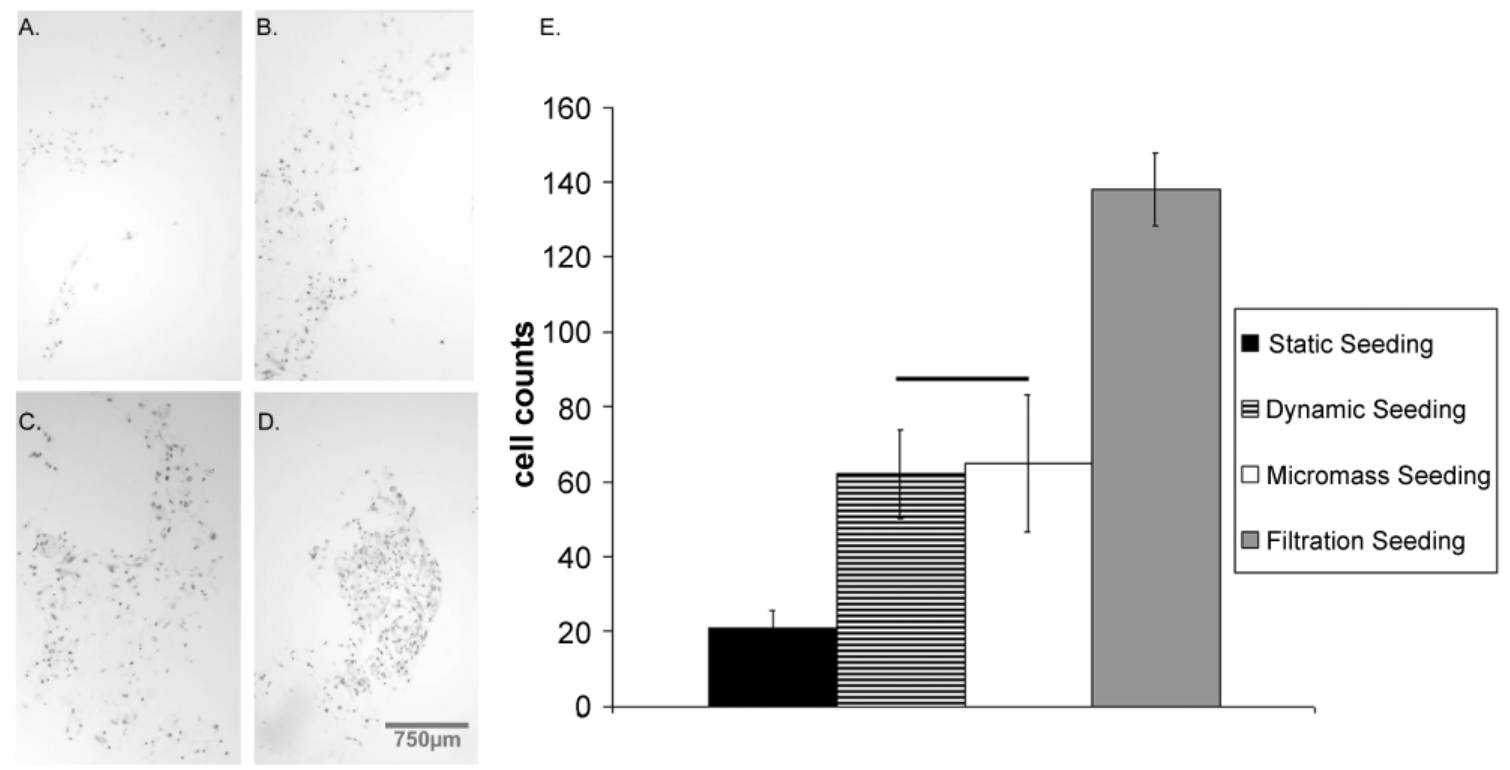

Figure 2: Cell count and distribution varies in seeded scaffolds, 6 hours after seeding. Histological slides demonstrate the even distribution and high cell adhesion produced in scaffolds that were seeded through filtration (A). Static seeding (B) is characterized by a lower yield of cells that are un-evenly allocated throughout the sections. Dynamic seeding showed sections with densely packed cells but also a large variation in cell location (C). Micromass seeding technique was validated showing a densely packed group of cells in the core of the scaffold. Quantification of the mean cell count (E) in histological demonstrates that filtration, dynamic, and micromass seeding techniques enable more cell adhesion $(\mathrm{p}<0.001)$. There was no significant difference between micromass and dynamic seeding $(\mathrm{p}=0.672)$. Bars indicate groups that are not significantly different.

Micromass seeding exhibited significantly higher cell counts than static seedingafter 6 hours in both mineralized and non-mineralized templates $(\mathrm{p}>0.001)$. Histology showed that micromass seeded scaffolds exhibited highly dense centralized localization of cells. Quantitatively,6 fold increase in the number of cells attached in filtration over static seeding, and validated dynamic seeding and micromass seeding as suitable seeding techniques that show significantly greater cell adhesion than static seeding $(\mathrm{p}<0.001$, for both over static seeding) (Figure 2E). The standard deviation in cell cluster numberis significantly lessin filtered seeding than the other methods (138.7+/-10.2 cells counts). Micromass seeding had the highest deviation (68.0+/-23.4 cells counts). Fibroblasts exhibited no significant difference from BMSCs in attachment (not shown). Because dynamic seeding was employed as a variation of filtration seeding and yielded lower cell retention, this technique was excluded from the rest of the biological experiments.

The presence of a mineralized scaffold had no effect in calcenin transfer. However, in mineralized scaffolds, transfer in filtered seeded cells and micromass seeded cells is only moderately significant $(\mathrm{p}=0.92)$. Furthermore, cells containing the gap junction inhibitor AGA, showed little transfer compared to both experimental groups without AGA $(\mathrm{p}<0.001)$ in both mineralized and PLGA scaffolds, indicating GJIC dependent transfer.

\section{Micromass seeded cultures enhance gap junction dependent cell-cell communication}

A significant increase in calcenin-AM transfer between donor and recipient cells in the micromass seeded cells after 5 hoursover filtered and static seeded cells ( $\mathrm{p}=0.034, \mathrm{p}<0.001$, respectively) (Figure 3 ).

\section{Seeding and template conditions alter bone marker expression}

Expression of bone differentiation markers ALP and OCN was significantly altered when different scaffolds and seeding techniques were implemented (Figure 4).

At day 2, ALP expression was significantly higher in micromass seeded than both filtration and static seeded scaffolds $(p<0.001$ for both), which exhibited no difference in the differentiation marker (Figure 4A). At day 8, micromass seeded scaffolds still exhibited significantly higher expression when compared to filtration and static seeded scaffolds $(\mathrm{p}<0.001$ for both), however, filtration also significantly expressed more ALP than static $(\mathrm{p}<0.001)$. At day 16 , 
Citation: Rosselló RA, Kohn DH (2014) Enhanced Volume of Stem Cell-Based Regenerated Tissue Achieved by Altering Microenvironment and Cell Seeding Strategies. J Tissue Sci Eng S: S:1. doi:10.4172/2157-7552.S1-005

Page 6 of 11

there was no significant difference in ALP expression between seeding

methods.

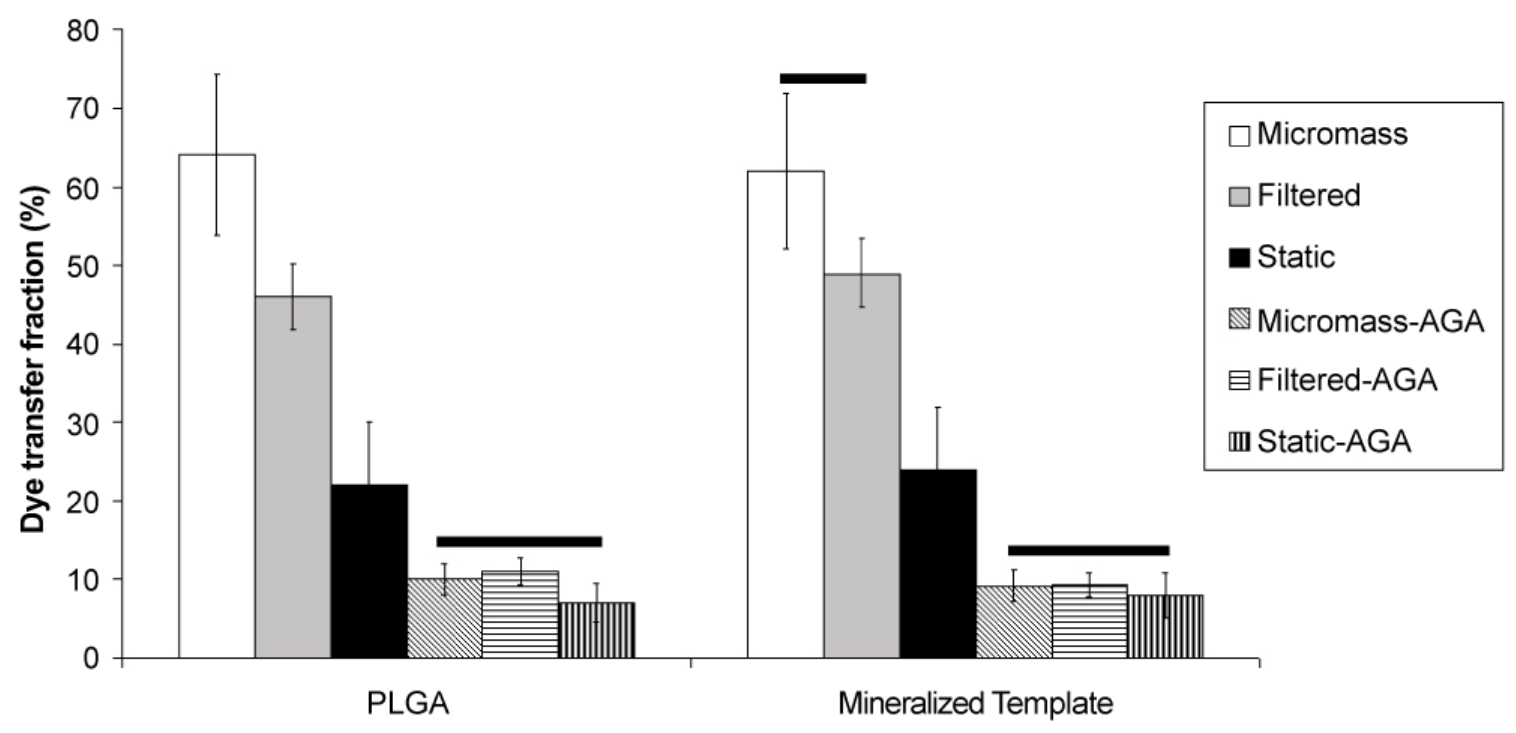

Figure 3: Seeding alters gap junction intercellular communication. Cells seeded in PLGA scaffolds by micromass transferred calcenin at a higher fraction $(63.2+/-10.6 \%)$ than both filtered $(46.2+/-5.4 \%)$ and static seeded cells $(23.6+/-6.9 \%)$. Although there was no significant differences in transfer between cells in mineralized and PLGA scaffolds (for all seeding strategies), micromass and filtered seeded cells exhibit no significant difference in transfer when seeded in a mineralized scaffold $(\mathrm{p}=9.2)$. Cells treated with AGA show significantly less transfer than those that were not, as well as showing no difference between seeding conditions exposed to the gap junction uncoupler. This indicates a gap junction dependent transfer of calcenin. Horizontal bars indicate groups that are not significantly different.

Expression of osteocalcin was different starting at day 8, where micromass seeding exhibited significantly higher expression $(\mathrm{p}<0.001$ for both) compared to filtration and static seeded scaffolds (Figure 4B). At day 16, OCN expression in micromass seeded scaffolds was significantly greater than filtration and static seeded scaffolds $(\mathrm{p}=0.0211)$. Cells seeded through filtration expressed significantly higher OCN than statically seeded cells $(\mathrm{p}<0.001)$.

Template mineralization and the presence of soluble calcium increased ALP and OCN expression over cells seeded in PLGA scaffolds (Figure 4A-4D). Cells seeded in mineralized templates by all seeding conditions expressed significantly larger amounts of ALP than cells seeded in PLGA scaffolds $(\mathrm{p}=0.021$ ) (Figure 4C and 4D). Increasing the concentration of soluble calcium to cells seeded in PLGA exhibited a significant increase when compared to cells seeded in PLGA. Filtered and static seeded scaffolds exhibited significant increases in expression $(\mathrm{p}=0.032, \mathrm{p}=0.042)$, while only moderately significant in micromass seeded cells $(\mathrm{p}=0.099)$. Fibroblast cells did not express ALP or Ostiocalcinin at detectable rates, after 38 cycles in qRTPCR, as was the case with the no-template control.

\section{Mineralized scaffolds enhance bone formation}

H\&E slides showed normal bone containing marrow that included fat, entrapped cells and hematopoietic cells for all groups seeded in mineralized scaffolds (Figure 5A-5E).
Static seeded scaffolds exhibit small bone formation with, but no marrow. In general, when a mineralized template is use, cells regenerate more bone (Figure 5A-5G). Bone formation is observed in the periphery of ossicles produced by filtered seeded cells, with increasing shell thickness in the mineralized scaffolds (Figure 5B and $5 \mathrm{E})$. Bone generated by micromass seeded scaffolds showed bone growth in the core of the ossicles and entrapped cells morphology indicative of bone tissue (Figure 5A and 5D). Statically seeded produced marginal bone formation in PLGA scaffolds and was clearly aided by the presence of a mineral layer in the scaffold (Figure 5C-5F).

Micromass and filtration seeding led to a higher BVF than static seeding (Figure 5G). There was no significant difference in BVF between the filtered $(27.3+/-2.5 \%)$ and micromass $(31.2+/-6.2 \%)$. However the percentage of variability was higher on the micromass seeded construct than the filtration. Filtered, micromass and statically seeded mineralized scaffolds showed significantly higher BVF ( $\mathrm{p}$ values $=0.013,0.037,0.009$, respectively) than the PLGA seeded counterparts. 
A.

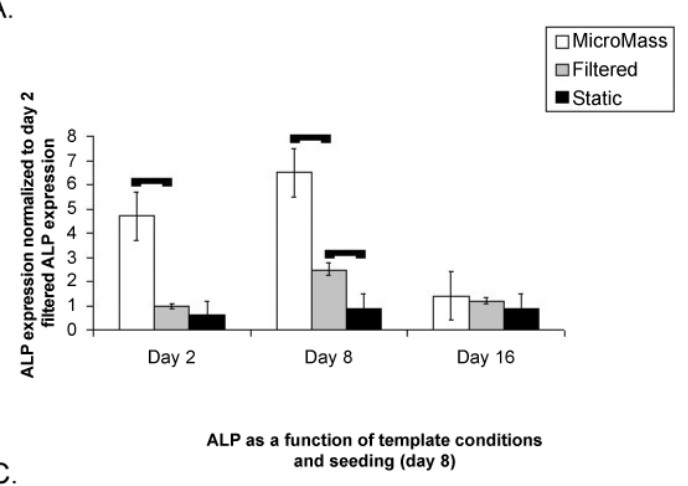

C.

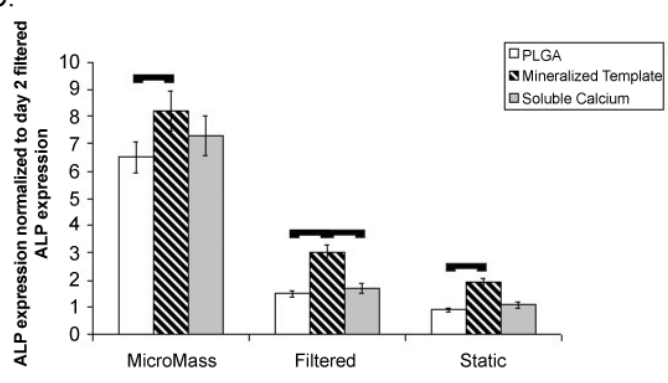

B.
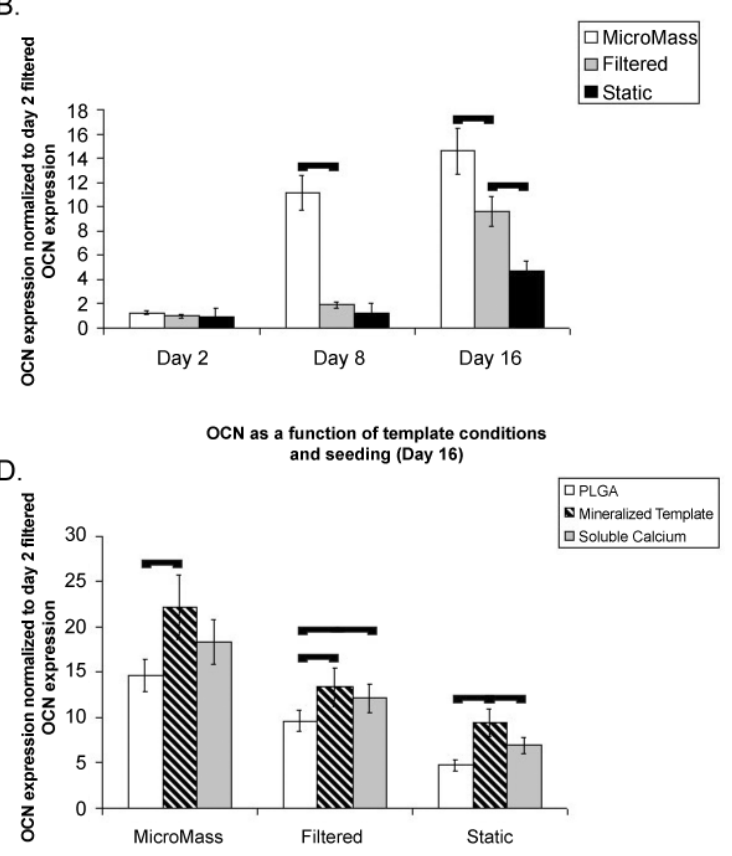

Figure 4: Expression of differentiation markers is increased with alternative seeding techniques and a mineralized template. Alkaline phosphatase (A) and Osteocalcin (B) expression increased significantly in cells seeded by micromass over filtration (ALP: $\mathrm{p}($ day 2$)<0.001$, $\mathrm{p}($ day 8$)<0.001 ;$ OCN: $\mathrm{p}($ day 8$)<0.001$, $\mathrm{p}($ day 16$)=0.0211)$ and static seeding ALP: $\mathrm{p}($ day2, day 8$)<0.001 ;$ OCN: $\mathrm{p}($ day 8 , day 16$)<0.001)$. Cells seeded in a mineralized template and calcium rich environment also expressed higher levels of ALP (C) and OCN (D) over cells seeded in PLGA. Cells seeded in mineralized templates expressed significantly more ALP and OCN in all seeding techniques over cells seeded in PLGAonly (ALP: $\mathrm{p}($ day2) $0.031, \mathrm{p}($ day 8$)<0.001 \mathrm{p}($ day 16$)<0.001 ; \mathrm{p}($ day2 $)<0.001 \mathrm{OCN}$ : $\mathrm{p}($ day 8$)=0.033$, $\mathrm{p}($ day 16$)<0.001)$. Although cells seeded with increased extracellular calcium did not exhibit a significant difference in ALP relative to those seeded in PLGA, it did exhibit significant differences in expression in OCN expression for filtered and static seeded cells ( $\mathrm{p}=0.032, \mathrm{p}=0.042$ respectively).

\section{Different seeding techniques led to distinct patterns of osteogenesis}

Distribution analysis performed on Von Kossa sections verified the qualitative observation in the H\&E sections, showing a quantitative difference in the distribution of mineral location in micromass and filtered ossicles (Figure 6A). The filtered ossicles showed most of the mineral in the periphery while the micromass ones had a more even distribution (Figure 6B).

The ossicles generated by filtration seeding showed significantly higher BVF in the periphery $(75-100 \%)$ than in the core $(0-25 \%$; $\mathrm{p}<0.001)$. The micromass seeded ossicles have significantly more mineral in the core $(p=0.0213)$, and significantly less $(p=0.0311)$ in the periphery than the filtered ones (Figure $6 \mathrm{~B}$ ). There was no significant difference in BVF between topographical regions in the ossicles generated by micromass seeding.

\section{Discussion}

Taken together, our data suggests that altering the initial cell seeding conditions in a biomimetic template, without interference of external or continuously sub-ministered agents can have a profound impact on both the amount and spatial distribution of regenerated tissue. Explicitly, we showed that mineralizing a polymer scaffold (PLGA) and seeding cells through filtration and micromass, as opposed to the conventionally used static seeding, enhanced differentiation and regeneration of bone in-vivo [15,35]. Furthermore, we showed that the filtration and micromass seeding enabled higher gap junction intercellular communication between cells in $3 \mathrm{D}$, an important component for tissue development and homeostasis [16-18,36]. Our results therefore providesimple alternatives that can have a significant impact in the regeneration of $3 \mathrm{D}$ tissue equivalents.

In our studies comparing mineralized and non-mineralized scaffolds, we investigated the effect of adhesion due to the biomimetic surface. Other studies have shown that such a calcium/phosphate apatite can enhance cell adhesion [37-39]. However, our results show that, only when statically seeded, cells adhered at higher rates in mineralized scaffolds over PLGA (Figure 2). Mineralized scaffolds seeded through micromass and filtration seeding exhibited similar rates of adhesion. The results observed in the statically seeded scaffolds are therefore consistent with experiments done by others, yet, coupled with our filtration data, suggests that the effect of adhesion is minimized when compared to altering the seeding strategy. Also, mineralized scaffolds did not have an effect on GJIC (Figure 3). This may be due the short term nature of the experiments, as other groups have shown that external influences by calcium can increase the GJIC in cells [21]. Future experiments should examine GJIC in longer term cultures, and investigate if the level of soluble and insoluble calcium enhances GJIC. 


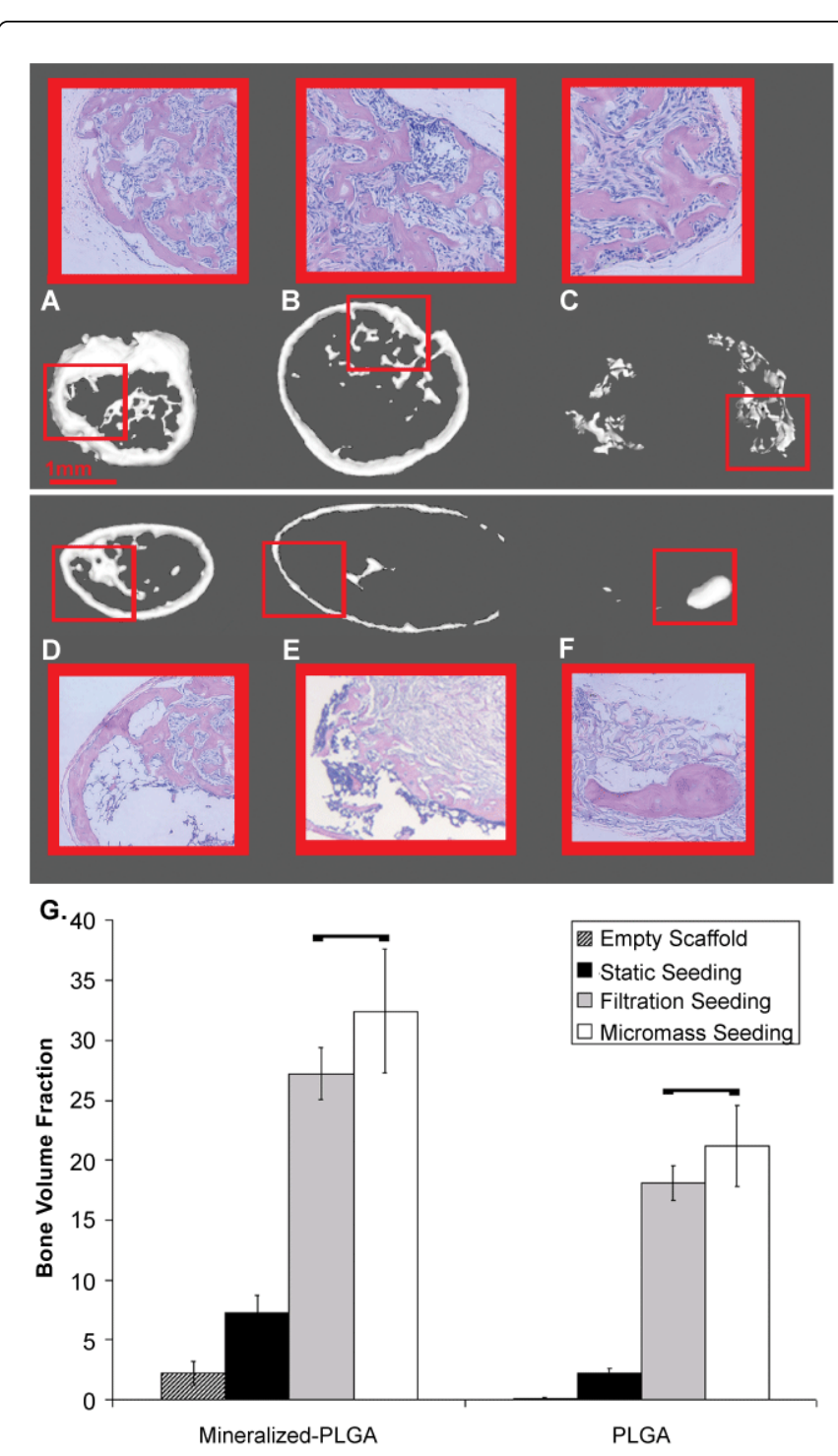

Figure 5: Volume fractions and patterns of osteogenesis vary as a function of scaffold surface and seeding techniques. Macromass seeded scaffolds produce smaller ossicles, with a more abundant mineralization in the core of the scaffold (A, D). Filtered scaffolds $(B, E)$ show a larger shell of bone formation with little or no mineralization in the core of the scaffold in both CT renderings and $\mathrm{H} \& \mathrm{E}$ histological sections. Static seeded scaffolds produce scant mineralization and bone formation $(\mathrm{C}, \mathrm{F})$. Mineralization enhances mineral coverage and BVF in all groups. These significant differences as a function of surface material and initial seedingwere quantified $(\mathrm{G})$. Both the micromass and filtration seeding yielded significantly larger BVF than static seeding $(\mathrm{p}<0.001)$. Although there was no significant difference between the filtration and macromass seeding, the variability is 2-fold greater in the macromass implants. Mineralized scaffolds showed a significantly higher BVF with all seeding techniques than did their PLGA counterpart $\quad(p$ (micromass) $0.037, \quad p($ filtration $)=0.013$, $\mathrm{p}($ static $)=0.009)$. Bars indicate groups that are not significantly different.
Mineralized templates had a significant effect both in cell differentiation in a 3D scaffold (Figure 4), and in the amount of regenerated bone in-vivo (Figure 5). The examination of differentiation under 3D conditions was important; recently, our group and others have demonstrated differences in cell differentiation between a $2 \mathrm{D}$ monolayer and $3 \mathrm{D}$ cultures $[1,44]$. The differentiation data contained a group that only contained soluble calcium. In these cases, there was moderate or significant increase in differentiation, relative to cells seeded in PLGA scaffolds. However, cells in mineralized templates, in general, had significantly higher differentiation marker expression than the soluble calcium group. This suggests that both soluble and insoluble calcium in these biomimetic scaffolds play an important role in differentiation. When cells were seeded in mineralized templates, the ossicles regenerated had a significantly higher BVF, relative to ossicles regenerated by cells seeded in PLGA. Perhaps the most significant effect is observed in the static seeded scaffolds, whereby the presence of a mineral layer, enables bone formation to occur, with entrapped cells in a peripheral shell formation, and marrow cavities forming, compared to partial sections of hard tissue with some entrapped cells observed in the polymer group. These results suggest that a mineralized layer provides a favorable physiological environment for cells to thrive, differentiate and regenerate tissue.

Altering seeding conditions had a significant impact in the number of adhered cells in culture, cell density, cell-to-cell communication, differentiation and patterns of osteogenesis. Filtration and micromass seeding showed significant increases in cell adhesion over static seeding. This result is promising, by constructs that may have other biologically favorable benefits, but are hard to seed due to their rigid nature. Filtration provides a mechanism for uniform and complete capacity seeding of a 3D structure (Figures 1 and 2), while micromass enables the targeted location of a dense cluster of cells. Cells seeded by filtration and micromass were analyzed for their capacity to engage in cell-to-cell communication, and compared to cells seeded statically. We chose to investigate this factor as intracellular communication through gap junctions is essential for proper development of tissues and homeostasis, specifically in bone $[40,41]$. The data clearly shows significant increases in GJIC, in both micromass and filtration seeded cells when compared to static seeded cells. This suggests that the higher cell density increases the formation of gap junctions between cells, enabling a higher grade of communication. Whether it is the increased proximity of cells or the increased stress experienced by the cells the predominant factor enhancing GJIC remains to be elucidated.

Differentiation of cells in a 3D constructs provide insights into the actual processes occurring within these scaffolds. The data showed enhanced differentiation due to the initial alteration in cell seeding. Specifically, cells seeded in micromasses exhibited early signs of differentiation, expressing high levels of ALP in the 2nd day after culture. Coupled with the early expression of the late differentiation marker, osteocalcin (Figure 4B), the results imply that micromass seeded cells prompts differentiation onset faster than the other methods. It is also evident that differentiation in filtered seeded cells was greater than in static seeded scaffolds (Figure $4 \mathrm{~A}$ and $4 \mathrm{~B}$ ), although different than cells seeded in micromasses. One explanation may be that cells in micromasses experience more nutrient and byproduct transport than the supersaturated cells in filtered scaffolds [7]. Although both have the benefits of high density, transport and cell migration may play a role in differentiation. 
A.

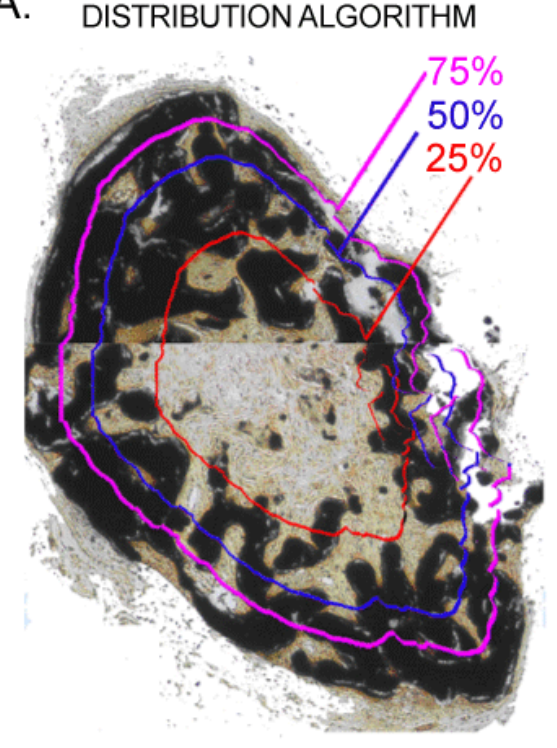

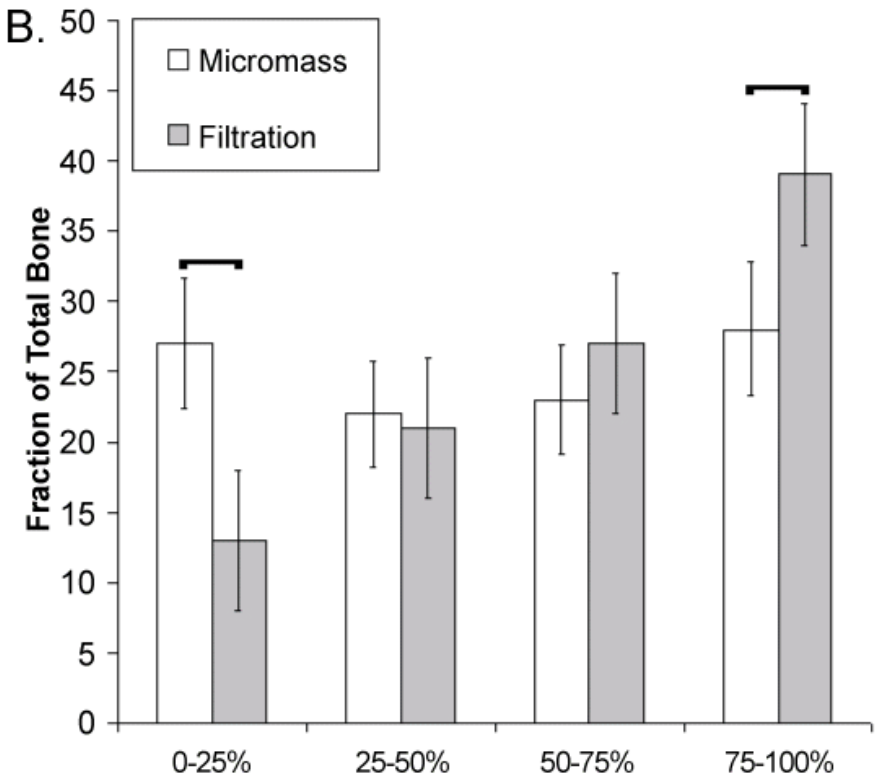

Figure 6: Topographic analysis of mineral distribution within bone ossicles. In order to quantify the observed differences in mineral distribution throughout the ossicles, cross sectional slides where analyzed with a program designed to calculate the topographical distribution of the mineral. The program was set to analyze sections in $25 \%$ increments from centroid (a). Even distribution of boneinossicles produced by micromass seeding showed no significant differences in bone \% between regions (b). Ossicles generated by scaffolds seeded through filtration showed a significant difference in mineral from the periphery $(75-100 \%)$ to the core $(0-25 \%) ; \mathrm{p}<0.001$. The amount of mineral content in $0-25$ th $\%$ of the micromassossicles was significantly greater than the filtered ossicles $(p=0.0213)$, while the opposite is true for the periphery $(\mathrm{p}=0.0311)$. Horizontal bars indicate groups that are significantly different.

Bone regeneration was also altered significantly as a function of seeding. Ossicles regenerated from filtered and micromass seeded cells, produced larger volumes of bone compared to static seeded cells (Figure 5). This result was particularly important when cells were seeded in PLGA scaffolds, as bone regenerated from statically seeded cells was characterized by sporadic bone formation, without marrow cavity or entrapped cells (Figure 5F). Not only did ossicles form from filtered and micromass seeded scaffolds with higher volumes of bone, but the distribution of tissue was significantly different in these two strategies. When seeded by filtration, cells produced a peripheral shell of bone, with diminished bone formation in the core of the ossicle. This may be due to cells thriving and differentiating in the periphery of the construct, while transport is increasingly impaired, thereby compromising tissue regeneration in the core of the scaffold. This contrasts the spatially distributed formation of bone that characterizes ossicles regenerated by micromass seeded cells. This increased presence of bone formation in micromass seeded ossicles may be due to both cell migration, nutrient transport and, a gradient of differentiation. Because cells are seeded in the core of the scaffold at high densities, and differentiation starts promptly, it enables formation of bone in the center. Cells may also migrate outward or inward from the body, and start differentiating at a later time, causing a differentiation gradient. The end result is a more spatially distributed and uniform tissue equivalent. The effect of migration in the extracellular matrix is a topic of intense research, and future studies in this field may look at the potential effects of migration in micromass seeded scaffolds $[42,43]$. With the generation of iPS cells, comes also potential generation of cancer cells and initial conditions of those cells and their distribution will be an important area of research [45]. The distribution studies in tandem with the overall analysis of the ossicles, strengthens the claim that altering the initial seeding conditions of cells can modify the amount and distribution of bone, and with future studies, such regeneration patterns may be achieved by design. Because fractures are a key objective in our regenerative scope, future studies will focus on performing these experiments in a bone defect model, in order to observe the generation of bone in-situ [46].

In conclusion, we showed that both a biomimetic template and the manipulation of initial seeding conditions can have profound effects on the resulting differentiation and in-vivo regeneration of bone. Biomimetic templates provided a physiologically favorable environment for BMSCs for tissue formation, while altering the seeding conditions in these rigid 3D scaffolds enabled higher cell adhesion, cell-to-cell communication, and larger volumes of bone with distinct patterns of regeneration. Altogether, our study addresses and provides a mechanism to solving the critical question of full tissue equivalent regeneration by showing that, with simple manipulations of the initial cell and template conditions, one can significantly enhance the regeneration and spatial distribution of tissue in-vivo; which has a major impact on bone regeneration and $3 \mathrm{D}$ tissue engineering as a whole.

\section{Acknowledgements}

The authors would like to thank the NIH grants R01 DE 015411 and DE013380 for supporting this work. 


\section{References}

1. Rosselló RA, Wang Z, Kizana E, Krebsbach PH, Kohn DH (2009) Connexin 43 as a signaling platform for increasing the volume and spatial distribution of regenerated tissue. ProcNatlAcadSci U S A 106: 13219-13224.

2. JanouskováO, Fales I, Kobylka P, Vonka V (2003) [Gene therapy of the graft versus host reaction]. CasLekCesk 142: 530-533.

3. Beaman FD, Bancroft LW, Peterson JJ, Kransdorf MJ (2006) Bone graft materials and synthetic substitutes. RadiolClin North Am 44: 451-461.

4. Hollister SJ, Maddox RD, Taboas JM (2002) Optimal design and fabrication of scaffolds to mimic tissue properties and satisfy biological constraints. Biomaterials 23: 4095-4103.

5. Taboas JM, Maddox RD, Krebsbach PH, Hollister SJ (2003) Indirect solid free form fabrication of local and global porous, biomimetic and composite 3D polymer-ceramic scaffolds. Biomaterials 24: 181-194.

6. Laurencin C, Khan Y, El-Amin SF (2006) Bone graft substitutes. Expert Rev Med Devices 3: 49-57.

7. Goldstein AS (2001) Effect of seeding osteoprogenitor cells as dense clusters on cell growth and differentiation. Tissue Eng 7: 817-827.

8. Goldstein AS, Juarez TM, Helmke CD, Gustin MC, Mikos AG (2001) Effect of convection on osteoblastic cell growth and function in biodegradable polymer foam scaffolds. Biomaterials 22: 1279-1288.

9. David H. Kohn, Kyungsup Shin, Sun-Ig Hong, A. ChampaJayasuriya, Elena V Leonova, et al. (2005) Self-Assembled Mineral Scaffolds as Model Systems for Biomineralization and Tissue Engineering. Proc 8th International ConferenceChemistry and Biology of Mineralized Tissues.

10. Holtorf HL, Sheffield TL, Ambrose CG, Jansen JA, Mikos AG (2005) Flow perfusion culture of marrow stromal cells seeded on porous biphasic calcium phosphate ceramics. Ann Biomed Eng 33: 1238-1248.

11. Katz RW, Hollinger JO, Reddi AH (1993) The functional equivalence of demineralized bone and tooth matrices in ectopic bone induction. J Biomed Mater Res 27: 239-245.

12. Ducheyne P, Qiu Q (1999) Bioactive ceramics: the effect of surface reactivity on bone formation and bone cell function. Biomaterials 20 : 2287-2303.

13. Cartmell SH, Porter BD, García AJ, Guldberg RE (2003) Effects of medium perfusion rate on cell-seeded three-dimensional bone constructs in vitro. Tissue Eng 9: 1197-1203.

14. Zhao F, Ma T (2005) Perfusion bioreactor system for human mesenchymal stem cell tissue engineering: dynamic cell seeding and construct development. BiotechnolBioeng 91: 482-493.

15. Burg KJ, Holder WD Jr, Culberson CR, Beiler RJ, Greene KG, et al. (2000) Comparative study of seeding methods for three-dimensional polymeric scaffolds. J Biomed Mater Res 51: 642-649.

16. Zhang S, Wu XY, Li YH, Xie LQ (2001) [Mechanotransduction in bone]. Space Med MedEng (Beijing) 14: 465-468.

17. Goldmann WH (2002) Mechanical aspects of cell shape regulation and signaling. Cell BiolInt 26: 313-317.

18. Jørgensen NR, Teilmann SC, Henriksen Z, Civitelli R, Sørensen OH, et al (2003) Activation of L-type calcium channels is required for gap junction-mediated intercellular calcium signaling in osteoblastic cells. J BiolChem 278: 4082-4086.

19. Willecke K, Eiberger J, Degen J, Eckardt D, Romualdi A, et al. (2002) Structural and functional diversity of connexin genes in the mouse and human genome. BiolChem 383: 725-737.

20. Murphy WL, Kohn DH, Mooney DJ (2000) Growth of continuous bonelike mineral within porous poly(lactide-co-glycolide) scaffolds in vitro. J Biomed Mater Res 50: 50-58.

21. Jørgensen NR, Henriksen Z, Brot C, Eriksen EF, Sørensen OH, et al. (2000) Human osteoblastic cells propagate intercellular calcium signals by two different mechanisms. J Bone Miner Res 15: 1024-1032.

22. Triggle DJ (2006) L-type calcium channels. Curr Pharm Des 12: 443-457.
23. Romanello M, Veronesi V, D'Andrea P (2003) Mechanosensitivity and intercellular communication in HOBIT osteoblastic cells: a possible role for gap junction hemichannels. Biorheology 40: 119-121.

24. Berridge MJ, Lipp P, Bootman MD (2000) The versatility and universality of calcium signalling. Nat Rev Mol Cell Biol 1: 11-21.

25. Romanello M, D'Andrea P (2001) Dual mechanism of intercellular communication in HOBIT osteoblastic cells: a role for gapjunctionalhemichannels. J Bone Miner Res 16: 1465-1476.

26. Røttingen J, Iversen JG (2000) Ruled by waves? Intracellular and intercellular calcium signalling. ActaPhysiolScand 169: 203-219.

27. Glicklis R, Shapiro L, Agbaria R, Merchuk JC, Cohen S (2000) Hepatocyte behavior within three-dimensional porous alginate scaffolds. BiotechnolBioeng 67: 344-353.

28. Nair LS, Bhattacharyya S, Laurencin CT (2004) Development of novel tissue engineering scaffolds via electrospinning. Expert OpinBiolTher 4: 659-668.

29. Leonova EV, Pennington KE, Krebsbach PH, Kohn DH (2006) Substrate mineralization stimulates focal adhesion contact redistribution and cell motility of bone marrow stromal cells. J Biomed Mater Res A 79: 263-270.

30. Liao CJ, Chen CF, Chen JH, Chiang SF, Lin YJ, et al. (2002) Fabrication of porous biodegradable polymer scaffolds using a solvent merging/ particulate leaching method. J Biomed Mater Res 59: 676-681.

31. Mikos AG, Thorsen AJ, Czerwonka LA, Bao Y, Langer R, et al. (1994) Preparation and characterization of poly(L-lactic acid) foams. Polymer 35: 1068-1077.

32. Murphy WL, Mooney DJ (2002) Bioinspired growth of crystalline carbonate apatite on biodegradable polymer substrata. J Am ChemSoc 124: 1910-1917.

33. Rossello RA, Wang Z, Krebsbach PH, Kohn DH (2005) Establishing micro CT thresholds for tissue engineered bone and comparison of bone regenerated from BMSC and transduced C-4 cells. Trans 31st Annual Meeting Soc for Biomat

34. Wallace JM, Rajachar RM, Chen XD, Shi S, Allen MR, et al. (2006) The mechanical phenotype of biglycan-deficient mice is bone- and genderspecific. Bone 39: 106-116.

35. Holy CE, Shoichet MS, Davies JE (2000) Engineering three-dimensional bone tissue in vitro using biodegradable scaffolds: investigating initial cell-seeding density and culture period. J Biomed Mater Res 51: 376-382.

36. Cotrina ML, Lin JH, Alves-Rodrigues A, Liu S, Li J, et al. (1998) Connexins regulate calcium signaling by controlling ATP release. ProcNatlAcadSci U S A 95: 15735-15740.

37. Yang Y, Bumgardner JD, Cavin R, Carnes DL, Ong JL (2003) Osteoblast precursor cell attachment on heat-treated calcium phosphate coatings. J Dent Res 82: 449-453.

38. Shu R, McMullen R, Baumann MJ, McCabe LR (2003) Hydroxyapatite accelerates differentiation and suppresses growth of MC3T3-E1 osteoblasts. J Biomed Mater Res A 67: 1196-1204.

39. Suzuki T, Yamamoto T, Toriyama M, Nishizawa K, Yokogawa Y, et al. (1997) Surface instability of calcium phosphate ceramics in tissue culture medium and the effect on adhesion and growth of anchorage-dependent animal cells. J Biomed Mater Res 34: 507-517.

40. Lecanda F, Warlow PM, Sheikh S, Furlan F, Steinberg TH, et al. (2000) Connexin43 deficiency causes delayed ossification, craniofacial abnormalities, and osteoblast dysfunction. J Cell Biol 151: 931-944.

41. Stains JP, Civitelli R (2005) Cell-to-cell interactions in bone. BiochemBiophys Res Commun 328: 721-727.

42. Semino CE, Kamm RD, Lauffenburger DA (2006) Autocrine EGF receptor activation mediates endothelial cell migration and vascular morphogenesis induced by VEGF under interstitial flow. Exp Cell Res 312: 289-298.

43. Zaman MH, Matsudaira P, Lauffenburger DA (2007) Understanding effects of matrix protease and matrix organization on directional persistence and translational speed in three-dimensional cell migration. Ann Biomed Eng 35: 91-100. 
Citation: Rosselló RA, Kohn DH (2014) Enhanced Volume of Stem Cell-Based Regenerated Tissue Achieved by Altering Microenvironment and Cell Seeding Strategies. J Tissue Sci Eng S: S:1. doi:10.4172/2157-7552.S1-005

Page 11 of 11

44. Rossello RA, H D (2010) Cell communication and tissue engineering CommunIntegrBiol 3: 53-56.

45. Rosselló RA, Chen CC, Dai R, Howard JT, Hochgeschwender U, et al (2013) Mammalian genes induce partially reprogrammed pluripotent stem cells in non-mammalian vertebrate and invertebrate species. Elife 2: e00036.
46. Rossello RA, Kohn DH. (2009) Gap junction intercellular communication: A review of a potential platform to modulate craniofacial tissue engineering. Journal of Biomedical Materials Research Part B: Applied Biomaterials 88B: 509-518. 